\title{
ON ZIMMERMANN-HUISGEN'S SPLITTING THEOREM
}

\author{
VICTOR CAMILLO
}

\begin{abstract}
This note is motivated by a paper of Birge Zimmermann-Huisgen, which in turn is motivated by a long sequence of papers-the first due to Faith-dealing with the question of when the canonical embedding of a direct sum of modules in the corresponding direct product splits. Zimmermann-Huisgen answered a question raised by previous authors by showing that if $R$ is a von Neumann regular ring the only way this can happen is that, except for a finite number, the modules involved must each be semisimple with only a finite number of simple modules involved.

Based on a new, more elementary argument, we establish a necessary condition for the sum-product splitting over an arbitrary (associative) ring $R$ (with identity).
\end{abstract}

This note is motivated by a paper of Birge Zimmermann-Huisgen [3], which in turn is motivated by a long sequence of papers - the first due to Faith [1] - dealing with the question of when the canonical embedding of a direct sum of modules in the corresponding direct product splits. Zimmermann-Huisgen answered a question raised by previous authors by showing that if $R$ is a von Neumann regular ring the only way this can happen is that, except for a finite number, the modules involved must each be semisimple with only a finite number of simple modules involved. See the text for a precise statement.

Based on a new, more elementary argument, we establish a necessary condition for the sum-product splitting over an arbitrary (associative) ring $R$ (with identity). The above-mentioned result is obtainable as an immediate consequence of

THEOREM 1. Let $\left(M_{i}\right)_{i \in I}$ be a family of right $R$-modules. If the canonical embedding $c: \sum_{i \in I} \oplus M_{i} \rightarrow \prod_{i \in I} M_{i}$ splits, then there is a cofinite subset $J$ of I such that the factor ring $R / \operatorname{ann}_{R} \prod_{i \in J} M_{i}$ has the ascending chain condition on annihilators.

In the special case where all the $M_{i}$ are equal, this is due to Zimmermann [4, Satz 6.2]. This, in turn, extends a previous result of Lenzing for $M_{i}=R$ for all $i[2$, Proposition 2].

We are able to prove this result by applying a lemma of Wolfgang Zimmermann [4]. I happily ackowledge that after the above theorem was proved, it was Birge Zimmermann-Huisgen who pointed out that the result could be obtained using this lemma, and the proof of my generalization as written in the text is in fact hers.

Received by the editors March 9, 1984 and, in revised form, July 5, 1984.

1980 Mathematics Subject Classification. Primary 16A64, 16A30; Secondary 16A52.

${ }^{1}$ This result was obtained while the author was in residence with the Carson and Barnes Circus. The author would like to thank the president, D. R. Miller, for his gracious hospitality and the opportunity for uninterrupted work.

(1985 American Mathematical Society $0002-9939 / 85 \$ 1.00+\$ .25$ per page 
Notation. All modules are right modules unless otherwise stated. Annihilators should be clear from the context.

A $p$-functor defined on $\operatorname{Mod} R$ is a subfunctor of the forgetful functor $F$ : $\operatorname{Mod} R \rightarrow \operatorname{Mod} Z$ that commutes with direct products. $U$ is a subfunctor of $F$ means that for every $R$-module $M$, there is associated an abelian subgroup $M U$ in such a way that $f(N U) \subset M U$ whenever $f: N \rightarrow M$ is a homomorphism. $U$ commutes with products means that $\left(\Pi M_{i}\right) U=\Pi\left(M_{i} U\right)$. A natural example is the following: Given any subset $A$ of $R$, defined $U$ via $M U=\operatorname{ann}_{M} A$.

A fundamental lemma connecting $p$-functors with the splitting of countable sums in products was proved by Wolfgang Zimmermann [4, Lemma 3.2]. Here $N$ is the set of natural numbers.

Lemma 2. Let $P=\prod_{i \in I} M_{i}, S=\sum_{i \in I} \oplus M_{i}$, and for every subset $K$ of $I$ write $P_{K}=\prod_{i \in K} M_{i}$. Moreover, let $\left\{U_{n} \mid n \in \mathbf{N}\right\}$ be a descending chain of p-functors. Then splitting of the canonical embedding $0 \rightarrow S \rightarrow P$ implies the existence of a cofinite subset $J$ of $I$ and a natural number $n_{0}$ such that

$$
P_{J} U_{n}=P_{J} U_{n_{0}} \text { for all } n \geqslant n_{0}
$$

(in other words, $M_{i} U_{n}=M_{i} U_{n_{0}}$ for all $n \geqslant n_{0}$ and all $i \in J$ ).

Proof of Theorem 1. Retaining the notation of the lemma, suppose that the canonical embedding $0 \rightarrow S \rightarrow P$ splits.

Step A. In the first step we establish the existence of a cofinite subset $J$ of $I$ such that, for each cofinite subset $K \subset J$, we have $\operatorname{ann}_{R}\left(P_{K}\right)=\operatorname{ann}_{R}\left(P_{J}\right)$; in other words, the set $\left\{\operatorname{ann}_{R}\left(P_{K}\right) \mid K \subset I\right.$ cofinite $\}$ contains a maximal element.

The contrary would mean that each cofinite subset $J$ of $I$ contains a decreasing chain $J \supset K_{1} \supset K_{2} \supset K_{3} \supset \cdots$ of cofinite subsets with $\operatorname{ann}_{R}\left(P_{K_{n+1}}\right) \supsetneqq \operatorname{ann}_{R}\left(P_{K_{n}}\right)$ for all $n$. Fix one chain and let $L=\bigcup_{n \in \mathrm{N}}\left(I-K_{n}\right)$; our first claim would fail for the countable split sum-product inclusion $0 \rightarrow \oplus_{i \in L} M_{i} \rightarrow \prod_{i \in L} M_{i}$. Therefore, we may again retreat to the case $I=\mathbf{N}$ and simplify the notation to $P_{n}=\prod_{i \geqslant n} M_{i}$.

Applying the lemma to the descending chain of $p$-functors $\left\{U_{n} \mid n \in \mathbf{N}\right\}$ with $M U_{n}=\operatorname{ann}_{M} \operatorname{ann}_{R}\left(P_{n}\right)$,we obtain an index $n_{0}$ such that $P_{n_{0}} U_{n}=P_{n_{0}} U_{n_{0}}$ for all $n \geqslant n_{0}$, that is,

$$
\operatorname{ann}_{P_{n_{0}}} \text { ann }_{R}\left(P_{n}\right)=\operatorname{ann}_{P_{n_{0}}} \operatorname{ann}_{R}\left(P_{n_{0}}\right) \text { for all } n \geqslant n_{0} \text {. }
$$

Since ann $\operatorname{ann}_{P_{n_{0}}} \operatorname{ann}_{R}(X)=\operatorname{ann}_{R}(X)$ for any subset $X \subset P_{n_{0}}$, we derive ann ${ }_{R}\left(P_{n}\right)$ $=\operatorname{ann}_{R}\left(P_{n_{0}}\right)$ for all $n \geqslant n_{0}$ as desired.

Step B. Let $J$ be a cofinite subset of $I$ such that $A=\operatorname{ann}_{R}\left(P_{J}\right)$ is maximal in the sense of Step A. We wish to show that $S=R / A$ has the ascending chain condition on right annihilators.

First notice that the canonical embedding $0 \rightarrow \bigoplus_{i \in J} M_{i} \rightarrow \prod_{i \in J} M_{i}=P_{J}$ splits as an embedding of $S$-modules and that, by construction, $P_{K}$ is a faithful $S$-module for every cofinite subset $K$ of $J$.

Next, given any descending chain $B_{1} \supset B_{2} \supset B_{3} \supset \cdots$ of subsets of $S$, apply the lemma to the product $P_{J}$ and the descending chain $\left\{V_{n} \mid n \in \mathbf{N}\right\}$ of $p$-functors on 
mod $S$ given by $M V_{n}^{\prime}=\operatorname{ann}_{M} \operatorname{ann}_{S} B_{n}$, where $a_{n n}$ denotes the right annihilator in $S$. This furnishes a cofinite subset $K$ of $J$ and a natural number $n_{0}$ such that

$$
P_{K} V_{n}=P_{K} V_{n_{0}} \text { for all } n \geqslant n_{0},
$$

or, more explicitly,

$$
\operatorname{ann}_{P_{K}} \operatorname{ann}_{S} B_{n}=\operatorname{ann}_{P_{K}} \text { ann }_{S} B_{n_{0}} \text { for all } n \geqslant n_{0} .
$$

But it is easy to see that if $X$ is any faithful $S$-module and $A \subset B$ are subsets of $S$, then ann $\operatorname{ann}_{S} A=\operatorname{ann}_{X} \operatorname{ann}_{S} B$ implies ann $A=\operatorname{ann}_{S} B$. In fact, we have $X B \subset \operatorname{ann}_{X}$ ann $_{S} B=\operatorname{ann}_{X}$ ann $_{S} A$, whence $A C=0$ entails $X B C=0$; but $X$ being faithful, $X B C=0$ is tantamount to $B C=0$. The special choice $X=P_{K}$ thus yields

$$
\operatorname{ann}_{S} B_{n}=\operatorname{ann}_{S} B_{n_{0}} \text { for all } n \geqslant n_{0} .
$$

COROllaRy 3 (ZIMmermanN - HUISGEN [3]). Let $R$ be a von Neumann regular ring and $\left\{M_{i} \mid i \in I\right\}$ a family of $R$-modules. Then the following statements are equivalent:

(1) The canonical embedding of the direct sum of the $M_{i}$ in the product splits.

(2) There is a cofinite subset $J$ of I such that $\sum_{i \in J} \oplus M_{i}$ is injective.

(3) There is a cofinite subset $J$ of I such that $\sum_{i \in J} \oplus M_{i}$ is semisimple with only finitely many homogeneous components, and with each of the occurring simple modules finitely generated over its endomorphism ring.

Proof. (1) $\Rightarrow(3)$. By the theorem there is a cofinite subset $J \subset I$ such that $R / A$ has the ascending chain condition on right annihilators, where $A=\operatorname{ann}_{R}\left(\prod_{i \in J} M_{i}\right)$. Since $R / A$ is von Neumann regular, this means that $R / A$ is semisimple, and, hence, as an $R / A$-module, $\sum_{i \in J} \oplus M_{i}$ has the claimed structure. But this structure immediately carries over to the analogous behavior of the $R$-module $\sum_{i \in J} \oplus M_{i}$.

(3) $\Rightarrow(2)$. This is due to Faith [1].

(2) $\Rightarrow$ (1). Trivial.

COROllary 4. Let $R$ be a simple ring. Suppose there is a split canonical sequence $0 \rightarrow \sum_{i=1}^{\infty} \oplus M_{i} \rightarrow \prod_{i=1} M_{i}$ with each $M_{i} \neq 0$. Then $R$ has the ascending chain condition on right annihilators.

Proof. Since $R$ is simple, ann $\left(\Pi M_{i}\right)=0$.

Question. If $R$ is simple and has a.c.c. on annihilators, is there a nontrivial splitting as above?

\section{REFERENCES}

1. C. F. Faith, Rings with ascending chain condition on annihilators, Nagoya Math. J. 27 (1966), 179-191.

2. H. Lenzing. Direct sums of modules as direct summands of their direct product, Comm. Algebra 4 (1976), 681-691.

3. B. Zimmermann-Huisgen, The sum-product splitting property and injective direct sums of modules over i'on Neumann regular rings, Proc. Amer. Math. Soc. 83 (1981), 251-254.

4. W. Zimmermann, Rein injektive direkte Summen von Moduln, Comm. Algebra 5 (1977), 1083-1117.

Dipartiment of Mathematics, University of Iowa, Iowa City, Iowa 52242 